\title{
Influência alelopática de hortelã (Mentha x villosa Huds.) sobre emergência de plântulas de alface (Lactuca sativa L.)
}

\author{
MAIA, J.T.L.S. ${ }^{*}$; BONFIM, F.P.G. ${ }^{1}$; BARBOSA, C.K.R. ${ }^{1}$; GUILHERME, D.O. ${ }^{3}$; HONÓRIO, I.C.G. ; MARTINS, \\ E.R. ${ }^{2}$ \\ ${ }^{1}$ Departamento de Fitotecnia, Universidade Federal de Viçosa, Avenida PH. Holfs, s/n, CEP: 36570-000, Viçosa- \\ Brasil `janinitatimaia@yahoo.com.br ${ }^{2}$ Instituto de Ciências Agrárias, Universidade Federal de Minas Gerais, \\ Avenida Universitária 1000, Bairro Universitário, Caixa Postal 135, CEP: 39.404-006, Montes Claros-Brasil \\ ${ }^{3}$ Universidade Estadual do Norte Fluminense Darcy Ribeiro, Avenida Alberto Lamego, 2000, Horto, CEP: 28013- \\ 602, Campos dos Goytacazes-Brasil
}

\begin{abstract}
RESUMO: Objetivou-se com esse trabalho avaliar a interferência de possíveis efeitos alelopáticos de Menthax villosa Huds. sobre a emergência de Lactuca sativa L. As atividades foram conduzidas em laboratório, no Instituto de Ciências Agrárias (ICA) da Universidade Federal de Minas Gerais, em Montes Claros/MG. Foram propostos três tratamentos, sendo esses: solo A, coletado em área cultivada com hortelã no horto medicinal do ICA/UFMG; solo B, coletado em áreas adjacentes e, testemunha (papel germiteste). O delineamento experimental foi inteiramente casualizado (DIC), com sete repetições. As variáveis avaliadas foram: porcentagem e índice de velocidade de emergência. Observou-se que a emergência de plântulas de Lactuca sativa foi significativamente afetada pelo solo $A(6,95 \%)$ se comparada com o solo $B(21,71 \%)$, representando assim, uma inibição da emergência, ou seja, efeito alelopático da hortelã sobre a emergência de alface. Já o índice de velocidade de emergência (IVE) não diferiu significativamente entre os solos testados.
\end{abstract}

Palavra-chave: alelopatia, germinação, plantas medicinais

\begin{abstract}
Allelopathic influence of mint (Mentha $x$ villosa Huds.) on the emergence of lettuce (Lactuca sativa L.) seedlings. This study aimed to assess the interference of possible allelopathic effects of Menthax villosa Huds. on the germination of Lactuca sativa L. The activities were carried out in laboratory, at the Institute of Agricultural Sciences (ICA) of the Federal University of Minas Gerais, Montes Claros, Minas Gerais State, Brazil. The following three treatments were proposed: soil A, collected in area cultivated with mint at the garden of ICA; soil B, collected in adjacent areas; and control (germitest paper). Experimental design was completely randomized (CR), with seven replicates. The evaluated variables were: germination percentage and speed index. The emergence of Lactuca sativa seedlings was significantly affected by soil A $(6.95 \%)$ when compared to soil B (21.71\%), indicating thus emergence inhibition, i.e. an allelopathic effect of mint on lettuce emergence. On the other hand, the germination speed index (GSI) did not differ significantly between the tested soils.
\end{abstract}

Key words: allelopathy, germination, medicinal plants

\section{INTRODUÇÃO}

A alelopatia é definida como o efeito direto ou indireto, que pode ser benéfico ou danoso, de uma planta sobre outra via produção de compostos químicos que são liberados no ambiente (Rice, 1984). Este conceito pode abranger o reino animal, porém, apesar de ser verificada entre todos os organismos, a alelopatia nas plantas é mais comum e evidente
(Goetze \& Thomé, 2004).

A ação alelopática ocorre por meio da interação entre as condições ambientais e o efeito dos metabólitos secundários (Iganci et al., 2006). Os monoterpenos, componentes majoritários dos óleos essenciais de um grande número de espécies, tem sido relatados como aleloquímicos eficazes pelo efeito

Recebido para publicação em 07/04/2009

Aceito para publicação em 19/12/2010

Rev. Bras. Pl. Med., Botucatu, v.13, n.3, p.253-257, 2011. 
tóxico sobre a germinação de sementes (Souza Filho et al., 2009), crescimento de bactérias (Arruda et al., 2006) e sobre o crescimento de alguns fungos patogênicos através do pré-planto (Hasse et al., 2007). Os monoterpenos podem causar extensivos danos às membranas e ao processo respiratório das células, uma vez que podem ser sequestrados pelas estruturas secretoras das mesmas (Gershenzon et al., 2000).

Dentre as plantas medicinais que contém monoterpenos na composição do óleo essencial, as espécies do gênero Mentha se destacam pelo potencial alelopático. A exposição aos compostos voláteis cânfora e mentol de Lavandula latifolia, Artemisia camphorata e Mentha piperita induziu uma maior abertura estomática e o efeito deletério sobre a cutícula foliar em Arabidopsis thaliana, demonstrando que as camadas lipofílicas da superfície foliar e o estômato são primariamente afetados pelo ataque alelopático dos monoterpenos (Schulz et al., 2007). O óleo essencial de Carum carvi L., Mentha spicata L., Origanum onites L. e Thymbra spicata L. demonstraram alto efeito inibitório sobre sementes de Alcea pallida Waldst. \& Kit., Amaranthus retroflexus L., Centaurea salsotitialis L., Raphanus raphanistrum L., Rumex nepalensis Spreng., Sinapis arvensis L. and Sonchus oleraceus L.) em baixas concentrações (Azirak \& Karaman, 2008).

Nos últimos vinte anos, tem ocorrido uma incorporação de tecnologia à olericultura com o objetivo de garantir a produtividade, retorno econômico, ofertar produtos com melhor qualidade $e$ que ofereçam menor impacto ao ambiente (Rezende et al., 2005). A alelopatia, além de exercer algum tipo de controle sobre determinadas espécies espontâneas, do ponto de vista agronômico, contribui para o estabelecimento de espécies que não sejam fortemente alelopáticas, e que favoreçam a obtenção de lavouras equilibradas, com reflexos satisfatórios sobre a produtividade e longevidade das mesmas (Wardle, 1987). Segundo Cecílio Filho \& May (2002), uma das técnicas que podem favorecer os novos rumos da agricultura é o cultivo consorciado de hortaliças. Porém, a escolha das condições da área e a forma de conduzir a cultura, e as espécies que irão compor o sistema podem alterar a produtividade (Heredia Zárate, 2005), uma vez que estas espécies exercem um a cooperação mútua, obtendo a utilização de todos os fatores de produção do meio, com benefícios máximos para as espécies envolvidas.

A resistência ou tolerância aos aleloquímicos é geralmente específica, pois existem plantas que são mais sensíveis que outras (Ferreira \& Áquila, 2000). A hortelã (Mentha $\mathrm{x}$ villosa Huds.), aparentemente exerce ação alelopática sobre plantas de alface (Lactuca sativa L.), pois se observa diminuição no crescimento desta hortaliça, quando consorciada com a hortelã (Maia et al., 2008), indicando a necessidade de investigação no sentido de avaliar o seu potencial alelopático. Portanto, objetivou-se com esse trabalho avaliar a interferência de possíveis efeitos alelopáticos de Menthax villosa sobre a emergência de Lactuca sativa.

\section{MATERIAL E MÉTODO}

As atividades de pesquisa foram conduzidas em laboratório, no Instituto de Ciências Agrárias (ICA) da Universidade Federal de Minas Gerais, em Montes Claros/MG. Foram propostos três tratamentos, sendo o solo A coletado em área cultivada com hortelã no horto medicinal do ICA/UFMG; solo B, coletado em áreas adjacentes e, a testemunha (papel germiteste). Foram feitas análises físico-químicas dos solos coletados que podem ser observados na Tabela 1.0 delineamento experimental utilizado foi o inteiramente casualizado (DIC) com sete repetições, 50 sementes por parcela. O teste foi realizado em câmara de germinação (B.O.D.) em caixas gerbox contendo 200 $\mathrm{g}$ de solo para cada parcela dos dois tratamentos e duas folhas de papel germiteste para a testemunha, sendo submetidas ao fotoperíodo de 14 horas (luz) à temperatura de $30^{\circ} \mathrm{C}$ e 10 horas (sem luz) à temperatura de $20^{\circ} \mathrm{C}$, simulando as condições de estação quente.

A metodologia adotada seguiu as recomendações das Regras para Análise de Sementes (Brasil, 1992). Para o umedecimento dos solos foram utilizados $80 \mathrm{~mL}$ de água destilada, já para o papel germiteste adicionou-se $8 \mathrm{~mL}$ com monitoramentos após sete dias. Optou-se pelo uso de alface, uma vez que seus parâmetros de germinação já foram estabelecidos, sendo a planta mais comum como espécie alvo para avaliar efeitos alelopáticos, devido tanto ao pequeno período de germinação (24 a 48 horas), quanto ao crescimento (Rodrigues, 2003). A resistência ou tolerância aos metabólitos secundários trata-se de uma característica espécie-específica, e para que a planta seja considerada como planta teste, deve apresentar germinação rápida e uniforme, e grau de sensibilidade, permitindo que os resultados sejam expressos sob baixas concentrações de substâncias alelopáticas (Ferreira \& Áquila, 2000).

As variáveis avaliadas foram porcentagem $e$ índice de velocidade de emergência. A porcentagem de emergência (PE) foi realizada através da contagem diária, no mesmo horário, do número de plântulas emergidas, durante o período de sete dias. O índice de velocidade de emergência (IVE) foi calculado utilizando a fórmula descrita por Edmond \& Drapala (1958), sendo a IVE $=\left[\left(N_{1} \times D_{1}\right)+\ldots+\left(N_{n} \times D_{n}\right)\right] /$ $\left(D_{1}+\ldots+D_{n}\right)$, em que, $N=$ número de plântulas emergidas em cada contagem e $\mathrm{D}=$ números de dia

Rev. Bras. PI. Med., Botucatu, v.13, n.3, p.253-257, 2011. 
TABELA 1. Caracterização química dos solos utilizados no experimento $(0-20 \mathrm{~cm})$.

\begin{tabular}{lcc}
\hline \multicolumn{1}{c}{ Características } & \multicolumn{2}{c}{ Solos } \\
\cline { 2 - 3 } & 7,8 & B \\
\hline $\mathrm{pH}$ em água & 36,2 & 6,9 \\
$\mathrm{P}$-remanescente $\left(\mathrm{mg} \mathrm{L}^{-1}\right)$ & 156 & 37,5 \\
$\mathrm{~K}\left(\mathrm{mg} \mathrm{dm}^{-3}\right)$ & 8,00 & 5,30 \\
$\mathrm{Ca}\left(\mathrm{cmol}_{\mathrm{c}} \mathrm{dm}^{-3}\right)$ & 1,00 & 1,00 \\
$\mathrm{Mg}\left(\mathrm{cmol}_{\mathrm{c}} \mathrm{dm}^{-3}\right)$ & 0,00 & 0,00 \\
$\mathrm{Al}\left(\mathrm{cmol}_{\mathrm{c}} \mathrm{dm}^{-3}\right)$ & 0,95 & 1,66 \\
$\mathrm{H}+\mathrm{Al}\left(\mathrm{cmol}_{\mathrm{c}} \mathrm{dm}^{-3}\right)$ & 9,40 & 6,56 \\
$\mathrm{Soma} \mathrm{de} \mathrm{bases}\left(\mathrm{cmol}_{\mathrm{c}} \mathrm{dm}^{-3}\right)$ & 9,40 & 6,56 \\
$\mathrm{t}\left(\mathrm{cmol}_{\mathrm{c}} \mathrm{dm}^{-3}\right)^{*}$ & 0 & 0 \\
$\mathrm{~m}(\%)^{*}$ & 10,35 & 8,22 \\
$\mathrm{~T}\left(\mathrm{cmol}_{\mathrm{c}} \mathrm{dm}^{-3}\right)^{*}$ & 91 & 80 \\
$\mathrm{~V}(\%)^{*}$ & 3,08 & 2,78 \\
$\mathrm{Matéria} \mathrm{orgânica}\left(\mathrm{dag} \mathrm{kg}^{-1}\right)$ &
\end{tabular}

* $\mathrm{t}$ - Capacidade de troca de cátions efetiva; $\mathrm{m}$ - Percentagem de saturação por alumínio; $\mathrm{T}$ - Capacidade de troca de cátions; $\mathrm{V}$ Percentagem de saturação de bases da CTC

da avaliação, no período de sete dias. Os dados da variável porcentagem de emergência foram transformados para arco seno $\sqrt{p e / 100}$, onde pe é a porcentagem de emergência.

\section{RESULTADO E DISCUSSÃO}

Através do resumo da análise de variância pode-se perceber que as variáveis determinadas (PE e IVE), apresentaram diferenças estatísticas em função dos tratamentos pelo Teste $\mathrm{F}$ a $1 \%$ de probabilidade (Tabela 2). Observou-se que a emergência de plântulas de Lactuca sativa foi significativamente afetada pelo solo A $(6,95 \%)$ se comparada com o solo B $(21,71 \%)$, representando assim, uma inibição da emergência. Ensaios como este, que resultam na diminuição da germinação de sementes de alface por plantas medicinais, também foram observados por Stein et al. (2004), que estudando a geminação e índice mitótico de sementes tratadas com extrato de tansagem (Plantago australis Lam.), verificaram que o aumento da concentração do extrato $\left(15,30\right.$ e $\left.60 \mathrm{mg} \mathrm{mL}^{-1}\right)$ resultou em efeito alelopático gradativo e crescente, causando a inibição da germinação (45; 28 e 9\%, respectivamente).

A testemunha permitiu $93,14 \%$ de emergência (Teste Tukey a $5 \%$ de probabilidade), conforme Tabela 3.
TABELA 2. Resumo da análise de variância das variáveis, porcentagem de emergência (PE) e velocidade de emergência (VE), da alface (Lactuca sativa L.) sob influência da hortelã (Menthax villosa Huds.).

\begin{tabular}{|c|c|c|c|}
\hline \multirow[t]{2}{*}{ FV } & \multirow[t]{2}{*}{$\mathbf{G L}$} & \multicolumn{2}{|c|}{ Quadrado Médio } \\
\hline & & $\mathrm{PE}$ & VE \\
\hline Tratamento & 2 & $2,92^{* *}$ & $195,95^{\star *}$ \\
\hline Resíduo & 18 & 0,40 & 2,13 \\
\hline Total & 20 & & \\
\hline C.V. (\%) & & 28,60 & 32,32 \\
\hline
\end{tabular}

TABELA 3. Valores médios para porcentagem de germinação (PE) e velocidade de germinação (IVE).

\begin{tabular}{cccc}
\hline Tratamentos & $\mathrm{PE}^{*}(\%)^{* *}$ & IVE \\
\hline $\begin{array}{c}\text { Sobre papel } \\
\text { (Testemunha) }\end{array}$ & $1,42(93,14 \%)$ & a & $10,56 \mathrm{a}$ \\
Solo A & $0,48(21,71)$ & b & $2,33 \mathrm{~b}$ \\
Solo B & $0,19(6,95 \%)$ & c & $0,68 \mathrm{~b}$ \\
\hline
\end{tabular}

As médias seguidas de pelo menos uma mesma letra minúscula na linha, não diferem significativamente entre si pelo teste de Tukey a $5 \%$ de probabilidade. * Dados transformados para $\operatorname{arcosseno} \sqrt{X / 100}$ de PG. ** Porcentagem de germinação. 
Os resultados são semelhantes aos encontrados por Alves et al. (2004), em que extratos voláteis de óleos essenciais de canela, alecrimpimenta, capim-citronela e alfavaca-cravo evidenciaram potencialidades alelopáticas inibitórias na germinação de sementes e comprimento da raiz da alface (Lactuca sativa). Souza et al. (2005) verificaram efeito dos extratos de plantas medicinais nativas do Rio Grande do Sul, sobre a germinação de sementes de alface, com resultados mais evidentes para as espécies Bauhnia candicans Benth., Luehea divaricata Mart. et. Zucc. e Lippia alba (Mill.) N.E.Brown. Ao contrário, Barreiro et al. (2005), avaliando o efeito alelopático de extratos de parte aérea de barbatimão (Stryphnodendron adstringens) na germinação e desenvolvimento em plântulas de pepino (Cucumis sativus), observaram que o efeito do extrato foi mais significativo no desenvolvimento da plântula de pepino do que na germinação. Isto está de acordo com Ferreira \& Borghetti (2004), que afirmam que o efeito alelopático pode não se dar sobre o percentual final de germinação, mas sim sobre a velocidade de germinação ou sobre outra etapa do processo.

O índice de velocidade de emergência (IVE) não diferiu significativamente entre os solos testados, sendo superior apenas na testemunha. Tal fato pode ser explicado quando se analisa a bioquímica das espécies, que de acordo com os processos enzimáticos podem se tornar resistentes em função da capacidade de degradação ou detoxificação celular, impedindo o efeito de substâncias inibitórias através da ativação de enzimas do metabolismo oxidativo (Almeida, 2006).

Os resultados indicaram ação alelopática de Menthax villosa sobre Lactuca sativa. É possível que os monoterpenos presentes nas espécies do gênero Mentha sejam os responsáveis pela alelopatia observada, sendo que os dados existentes na literatura confirmam esse fato. Os óleos essências das plantas Origanun syriacum, Micromeria fruticosa e Cymbopogon citratus $\mathrm{L}$. demonstraram atividade inibitória, em baixas concentrações, sobre a germinação de Amarantus blitioides, Amaranthus palmeri, Euphorbia hirta L., Sinaps nigra, Trifolium campestri e Lycopersicum esculentum L. (Dudai et al., 1999). Mentofurano, pulegol, neomentol, mentol e mentona afetaram o potencial de membrana em Cucumis sativus L. (Maffei et al., 2001).

O óleo essencial de Mentha piperita L. inibiu $50 \%$ a respiração radicular e mitocondrial em Cucumbis sativus L. sendo o pulegol o composto mais tóxico (Mucciarelli et al., 2001). Um grupo de sete plantas, incluindo Menta pulegium L. foi selecionado por afetar a germinação de Lactuca sativa (Shiraishi et al., 2002). Em seguida os efeitos dos extratos a partir de folhas secas e exsudatos das raízes dessas plantas foram analisados em agar. Entretanto, somente Phlox subulata L., Oxalis brasiliensis Lodd., Lycoris radiata Herbert. e Thymus serpyllum $\mathrm{L}$. inibiram o alongamento radicular de Amaranthus lividus L., Digitaria ciliaris (Retz.) Koel. e Chenopodium album L.

Investigações mais amplas, incluindo outras metodologias experimentais, principalmente testes em condições de campo, que evidenciem os efeitos de espécies com potencial alelopáticos são necessárias. Tais estudos tornam-se de extrema importância, uma vez que a utilização destas espécies pode contribuir na inibição de plantas espontâneas e na determinação de manejos mais adequados (Carvalho et al., 1996).

\section{CONCLUSÃO}

A emergência de plântulas de alface foi significativamente afetada pelo solo $\mathrm{A}$ (coletado em área cultivada com hortelã) quando comparada com o solo $B$ (coletado em áreas adjacentes). $O$ índice de velocidade de emergência (IVE) não diferiu significativamente entre os solos testados.

\section{AGRADECIMENTO}

À Coordenação de Aperfeiçoamento de Pessoal de Nível Superior (Capes) e à Fundação de Amparo a Pesquisa do Estado de Minas Gerais (Fapemig), pelo suporte financeiro.

\section{REFERÊNCIA}

ALMEIDA, L.F.R. Composição química e atividade alelopática de extratos foliares de Leonurus sibiriucs L. (Lamiaceae). 2006. 107p. Tese (Doutorado) - Instituto de Biociências, Universidade Estadual Paulista, Botucatu. ALVES, M.C.S. et al. Alelopatia de extratos voláteis na germinação de sementes e no comprimento da raiz de alface. Pesquisa Agropecuária Brasileira, v.39, n.11, p.1083-6, 2004.

ARRUDA, T.A. et al. Preliminary study of the antimicrobial activity of Mentha x villosa Hudson essential oil, rontundifolone and its analogues. Revista Brasileira de Farmacognosia, v.16, n.3, p.307-11, 2006.

AZIRAK, S.; KARAMAN, S. Alleopathic effect of some essential oils and components on germination of weed species. Acta Agriculturae Scandinavica, v.58, n.1, p.8892, 2008.

BARREIRO, A.P.; DELACHIAVE, M.E.A.; SOUZA, F.S. Efeito alelopático de extratos de parte aérea de barbatimão [Stryphnodendron adstringens (Mart.) Coville] na germinação e desenvolvimento da plântula de pepino. Revista Brasileira de Plantas Medicinais, v.8, n.1, p.48, 2005.

BRASIL. Ministério da Agricultura e Reforma Agrária. Regras para análise de sementes. Brasília: Secretaria Nacional de Defesa Agropecuária, 1992. 365p.

Rev. Bras. Pl. Med., Botucatu, v.13, n.3, p.253-257, 2011. 
CARVALHO, G.J. et al. Potencialidades alelopáticas de folhas verdes de cana-de-açúcar em diferentes concentrações de matéria seca na germinação de sementes de alface. Ciências, v.5, n.2, p.19-24, 1996. CECÍLIO FILHO, A.B.; MAY, A. Produtividade das culturas de alface e rabanete em função da época de estabelecimento do consórcio. Horticultura Brasileira, v.20, n.3, p.501-4, 2002.

DUDAI, N. et al. Essential oils as allelochemicals and their potential use as bioherbicides. Journal of Chemical Ecology, v.25, p.1079-89, 1999.

EDMOND, J.B.; DRAPALA, W.J. The effects of temperature, sand and soil, and acetone on germination of okra seed. Proceedings of the Americam Society Horticultural Science, v.71, p.428-34, 1954.

FERREIRA, A.G.; ÁQUILA, M.E.A. Alelopatia: uma área emergente da ecofisiologia. Revista Brasileira de Fisiologia Vegetal, v.12, n. esp., p.175-204, 2000. FERREIRA, A.G.; BORGHETTI, F. Germinação: do básico ao aplicado. Porto Alegre: Artmed, 2004. 520p.

GERSHENZON, J.; McCONKEY, M.E.; CROTEAU, R.B. Regulation of monoterpene accumulation in leaves of peppermint. Plant Physiology, v.122, p.205-13, 2000.

GOETZE, M.; THOMÉ, G.C.H. Efeito alelopático de extratos de Nicotiana tabacum e Eucalyptus grandis sobre a germinação de três espécies de hortaliças. Revista Brasileira de Agroeciência, v.10, n.1, p.43-50, 2004. HASSE, I.; MAY-DE MIO, L.L.; LIMA NETO, V.C. Efeito do pré-plantio com plantas medicinais e aromáticas no controle de Plasmodiophora brassicae. Summa Phytopathologica, v.33, n.1, p.74-9, 2007.

HEREDIA ZÁRATE, N.A. Propagação e tratos culturais em inhame (Colocasia esculenta (L.) Schott) cultivado em solo seco. In: ENCONTRO NACIONAL SOBRE A CULTURA DO INHAME, 2., 2005, Campo Grande. Anais... Campo Grande: UFMS, 2005. p.59-96.

IGANCI, J.R.V. et al. Efeito do extrato aquoso de diferentes espécies de boldo sobre a germinação e índice mitótico de Allium cepa L. Arquivos do Instituto Biológico, v.73, n.1, p.79-82, 2006.

MAFFEI, M.; CAMUSSO, W.; SACCO, S. Effect of Menthax piperita essential oil and monoterpens on cucumber root membrane potential. Phytochemistry, v.18, n.5, p.7037, 2001.
MAIA, J.T.L.S. et al. Produção de alface e cenoura em cultivo solteiro e consorciado com manjericão e hortelã. Revista Brasileira de Agroecologia, v.3, n.1, p.58-64, 2008.

MUCCIARELLI, M. et al. Effect of (+)-pulegone and other oil components of Mentha $x$ piperita on cucumber respiration. Phytochemistry, v.57, n.1, p.91-8, 2001.

REZENDE, B.L.A. et al. Viabilidade econômica das culturas de pimentão, repolho, alface, rabanete e rúcula e cultivo consorciado, na primavera-verão, Jaboticabal, Estado de São Paulo. Informações Econômicas, v.35, n.3, p.22-37, 2005.

RICE, E.L. Allelopathy. 2.ed. New York: Academic Press, 1984. 353p.

RODRIGUES, M.T.T. Empleo de los ensayos con plantas en el control de contaminantes tóxicos ambientales. Revista Cubana de Higiene y Epidemiología, v.41, n.3, p.2-3, 2003.

SCHULZ, M. Allelopathic monoterpenes interfere with Arabidopsis thaliana cuticular waxes and enhance transpiration. Plant Signaling \& Behavior, v.2, n.4, p.2319, 2007.

SHIRAISHI, S. Allelopathic activity of leaching from dry leaves and exudate from roots of ground cover plants assayed on agar. Weed Biology and Management, v.2, n.3, p.133-42, 2002.

SOUZA FILHO, A.P.S. et al. Efeitos potencialmente alelopáticos dos óleos essenciais de Piper hispidinervium C. DC. e Pogostemon heyneanus Benth sobre plantas daninhas. Acta Amazonica, v.39, n.2, p.389-96, 2009.

SOUZA, S.A.M. et al. Efeito de extratos aquosos de plantas medicinais nativas do Rio Grande do Sul sobre a germinação de sementes de alface. Revista Publicatio UEPG Ciências Biológicas e da Saúde, v.11, n.3, p.2938, 2005.

STEIN, V.C. et al. Germinação e índice mitótico de sementes tratadas com extrato de Plantago australis Lam. In: CONGRESSO DE INICIAÇÃO CIENTÍFICA E ENCONTRO DE PÓS-GRADUAÇÃO, 13., 2004, PelotasRS. Anais... Pelotas: UFPeL, 2004.

WARDLE, D.A. Allelopahtic in New Zealand pasture grassland ecosystem. Journal of Experimental Agriculture, v.15, p.243-55, 1987. 